\title{
An algorithm for closed-loop data-driven simulation
}

\author{
Ivan Markovsky* \\ * School of Electronics and Computer Science, University of Southampton \\ SO17 1BJ, United Kingdom, Email: im@ecs . soton . ac.uk
}

\begin{abstract}
Closed-loop data-driven simulation refers to the problem of constructing trajectories of a closed-loop system directly from data of the plant and a representation of the controller. Conditions under which the problem has a solution are given and an algorithm for computing the solution is presented. The problem formulation and its solution are in the spirit of the deterministic identification algorithms, i.e., in the theoretical analysis of the method, the data is assumed exact (noise free).
\end{abstract}

Keywords: Simulation, system identification, behaviors.

\section{PRELIMINARIES AND NOTATION}

We use the behavioural language, see Willems (1986). A discrete-time dynamical system with $\mathrm{w}$ manifest variables is a subset of the signal space $\left(\mathbb{R}^{\mathrm{w}}\right)^{\mathbb{N}}$, i.e., the set of functions from the set of natural numbers $\mathbb{N}$ to $\mathbb{R}^{\mathrm{w}}$. We assume that the manifest variables $w$ have a given input/output partition

$$
w=\operatorname{col}(u, y):=\left[\begin{array}{l}
u \\
y
\end{array}\right],
$$

where $u \in\left(\mathbb{R}^{\mathrm{m}}\right)^{\mathbb{N}}$ is an input and $y \in\left(\mathbb{R}^{\mathrm{p}}\right)^{\mathbb{N}}$ is an output.

The feedback interconnection of the plant $\mathscr{B} \subseteq\left(\mathbb{R}^{\mathrm{m}+\mathrm{p}}\right)^{\mathbb{N}}$ and a controller $\mathscr{C} \subseteq\left(\mathbb{R}^{\mathrm{r}+\mathrm{p}+\mathrm{m}}\right)^{\mathbb{N}}$

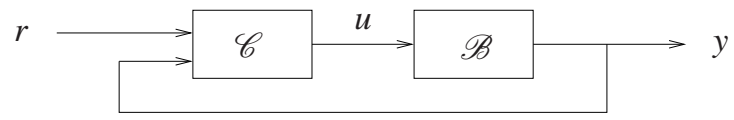

is given by

$$
\mathscr{B}_{\mathscr{C}}=\mathscr{B}_{\text {ext }} \cap \mathscr{C}
$$

where

$$
\mathscr{B}_{\text {ext }}:=\left\{(r, w) \in\left(\mathbb{R}^{\mathrm{r}+\mathrm{m}+\mathrm{p}}\right)^{\mathbb{N}} \mid w \in \mathscr{B}\right\} .
$$

We consider linear, time-invariant, and finite dimensional plants and controllers. A kernel representation $R(\sigma) w=0$, were $\sigma$ is the backwards shift operator

$$
\sigma w(t):=w(t+1)
$$

is parameterized by the polynomial matrix $R \in \mathbb{R}^{\mathrm{p} \times(\mathrm{m}+\mathrm{p})}[z]$, and an image representation $w=M(\sigma) g$ is parameterized by the polynomial matrix $M \in \mathbb{R}^{(\mathrm{m}+\mathrm{p}) \times \mathrm{m}}[z]$.

The Hankel matrix with $t$ block rows, composed of the signal $w \in\left(\mathbb{R}^{\mathrm{w}}\right)^{T}$ is denoted by

$$
\mathscr{H}_{t}(w):=\left[\begin{array}{cccc}
w(1) & w(2) & \cdots & w(T-t+1) \\
w(2) & w(3) & \cdots & w(T-t+2) \\
w(3) & w(4) & \cdots & w(T-t+3) \\
\vdots & \vdots & & \vdots \\
w(t) & w(t+1) & \cdots & w(T)
\end{array}\right]
$$

The signal $u=(u(1), \ldots, u(T))$ is called persistently exciting of order $L$ if the Hankel matrix $\mathscr{H}_{L}(u)$ is of full row rank.

The banded upper-triangular Toeplitz matrix with $t$ blockcolumns, related to the polynomial $r \in \mathbb{R}^{1 \times \mathrm{r}}[z], \operatorname{deg}(r)=: n$ is denoted by

$$
\mathscr{T}_{t}(r):=\left[\begin{array}{ccccccc}
r_{0} & r_{1} & \cdots & r_{n} & 0 & \cdots & 0 \\
0 & r_{0} & r_{1} & \cdots & r_{n} & \ddots & \vdots \\
\vdots & \ddots & \ddots & \ddots & \ddots & 0 \\
0 & \cdots & 0 & r_{0} & r_{1} & \cdots & r_{n}
\end{array}\right]
$$

\section{CLOSED-LOOP DATA-DRIVEN SIMULATION}

The data-driven simulation problem is defined in Markovsky et al. (2005), where its applications in subspace system identification Van Overschee and De Moor (1996); Markovsky et al. (2006) are presented. Its fundamental role in data-driven control is shown in Markovsky and Rapisarda (2007, 2008). This paper further develops the concept of data-driven simulation to closed-loop data-driven simulation, defined as follows.

Problem 1. (Closed-loop data-driven simulation). Given

- trajectory $w_{\mathrm{d}}=\left(w_{\mathrm{d}}(1), \ldots, w_{\mathrm{d}}(T)\right) \in\left(\mathbb{R}^{\mathrm{w}}\right)^{T}$ of an LTI system $\mathscr{B} \subset\left(\mathbb{R}^{\mathrm{w}}\right)^{\mathbb{N}}$, with an input/output partition $w=$ $(u, y) \in \mathscr{B}, u \in\left(\mathbb{R}^{\mathrm{m}}\right)^{\mathbb{N}}$ input, $y \in\left(\mathbb{R}^{\mathrm{p}}\right)^{\mathbb{N}}$ output;

- LTI controller $\mathscr{C} \subset\left(\mathbb{R}^{\mathrm{r}+\mathrm{p}+\mathrm{m}}\right)^{\mathbb{N}}$, with an input/output partition $(r, u, y) \in \mathscr{C}, r \in\left(\mathbb{R}^{\mathrm{r}}\right)^{\mathbb{N}}, y \in\left(\mathbb{R}^{\mathrm{p}}\right)^{\mathbb{N}}$ inputs, $u \in\left(\mathbb{R}^{\mathrm{m}}\right)^{\mathbb{N}}$ output; and

- reference signal $r_{\mathrm{r}}=\left(r_{\mathrm{r}}(1), \ldots, r_{\mathrm{r}}\left(T_{\mathrm{r}}\right)\right) \in\left(\mathbb{R}^{\mathrm{r}}\right)^{T_{\mathrm{r}}}$

find the set of responses $w_{\mathrm{r}}$ of the closed-loop system $\mathscr{B}_{\mathscr{C}}$ to the reference signal $r_{\mathrm{r}}$.

\section{Solution and computational algorithm}

For given $w_{\mathrm{d}}, \mathscr{C}, r_{\mathrm{r}}$, we aim to compute the signals $w_{\mathrm{r}}$, such that

$$
\left(r_{\mathrm{r}}, w_{\mathrm{r}}\right) \in \mathscr{B}_{\mathscr{C}} \quad \Longleftrightarrow \quad\left\{\begin{array}{l}
w_{\mathrm{r}} \in \mathscr{B} \\
\left(r_{\mathrm{r}}, w_{\mathrm{r}}\right) \in \mathscr{C}
\end{array}\right.
$$

Assumption that $\mathscr{B}$ is controllable, there is $M \in \mathbb{R}^{\mathrm{w} \times \mathrm{m}}[z]$, such that

$$
\mathscr{B}=\text { image }(M(\sigma)) \text {. }
$$

Consider a kernel representation of the controller

$$
\mathscr{C}=\{(r, w) \mid R(\sigma) \operatorname{col}(r, w)=0\} .
$$


In terms of the image and kernel representations of the plant and controller, (1) becomes

$$
\left\{\begin{array}{l}
\exists g, \text { s.t. } w_{\mathrm{r}}=M(\sigma) g \\
R(\sigma) \operatorname{col}\left(r_{\mathrm{r}}, w_{\mathrm{r}}\right)=0 .
\end{array}\right.
$$

We assume that the controller $\mathscr{C}$ is specified by a kernel representation, however, the plant $\mathscr{B}$ is only implicitly specified by the trajectory $w_{\mathrm{d}}$. In order to avoid using a representation of $\mathscr{B}$, we solves (2) for $w_{\mathrm{r}}$, replacing the first equation by

$$
w_{\mathrm{r}}=\mathscr{H}_{T_{\mathrm{r}}}\left(w_{\mathrm{d}}\right) g \text {. }
$$

The equivalence of $w_{\mathrm{r}}=M(\sigma) g$ and (3) holds under the following assumptions:

(1) the system $\mathscr{B}$ is controllable,

(2) the input component $u_{\mathrm{d}}$ of $w_{\mathrm{d}}$ is persistently exciting of order $T_{\mathrm{r}}+\mathbf{n}(\mathscr{B})$, where $\mathbf{n}(\mathscr{B})$ is the order of $\mathscr{B}$,

see (Markovsky et al., 2006, Section 8.4) and (Willems et al., 2005, Theorem 1). Therefore, under assumptions 1 and 2, the set of solutions $w_{\mathrm{r}}$ of the linear system of equations

$$
\begin{aligned}
& w_{\mathrm{r}}=\mathscr{H}_{T_{\mathrm{r}}}\left(w_{\mathrm{d}}\right) g \\
& \mathscr{T}_{T_{\mathrm{r}}}(R) \operatorname{col}\left(r_{\mathrm{r}}, w_{\mathrm{r}}\right)=0
\end{aligned}
$$

is equal to the set of trajectories $w_{\mathrm{r}}$ solving the closed-loop datadriven simulation problem.

Let $R=:\left[\begin{array}{ll}R_{r} & R_{w}\end{array}\right]$, where $R_{r} \in \mathbb{R}^{1 \times \mathrm{r}}[z]$ and $R_{w} \in \mathbb{R}^{1 \times \mathrm{w}}[z]$.

$$
\mathscr{T}_{T_{\mathrm{r}}}(R) \operatorname{col}\left(r_{\mathrm{r}}, w_{\mathrm{r}}\right)=0 \Longrightarrow \mathscr{T}_{T_{\mathrm{r}}}\left(R_{w}\right) w_{\mathrm{r}}=-\mathscr{T}_{T_{\mathrm{r}}}\left(R_{r}\right) r_{\mathrm{r}} .
$$

Substituting $w_{\mathrm{r}}=\mathscr{H}_{T_{\mathrm{r}}}\left(w_{\mathrm{d}}\right) g$ into (5) gives the following system of equations

$$
\underbrace{\mathscr{T}_{T_{\mathrm{r}}}\left(R_{w}\right) \mathscr{H}_{T_{\mathrm{r}}}\left(w_{\mathrm{d}}\right)}_{A} g=\underbrace{-\mathscr{T}_{T_{\mathrm{r}}}\left(R_{r}\right) r_{\mathrm{r}}}_{b}
$$

The matrix $A$ is $T_{\mathrm{r}} \times(\mathrm{r}+\mathrm{w}) T_{\mathrm{r}}$, so that the system $A g=b$ is underdetermined. Let $g_{0}$ be a particular solution, e.g., the leastnorm solution $g_{0}=A^{\dagger} b$ and let $N$ be a matrix whose columns span the null space of $A$. The set of solution of (4) for $g$ is

$$
\mathscr{G}:=\left\{g_{0}+N z \mid z \in \mathbb{R}^{\operatorname{col} \operatorname{dim}(N)}\right\} .
$$

Then the set of responses $w_{\mathrm{r}}$ of the closed-loop system $\mathscr{B}_{\mathscr{C}}$ to the reference signal $r_{\mathrm{r}}$ is

$\mathscr{W}_{\mathrm{r}}=\mathscr{H}_{T_{\mathrm{r}}}\left(w_{\mathrm{d}}\right) \mathscr{G}=\{\underbrace{\mathscr{H}_{T_{\mathrm{r}}}\left(w_{\mathrm{d}}\right) g_{0}}_{w_{\mathrm{r}, 0}}+\mathscr{H}_{T_{\mathrm{r}}}\left(w_{\mathrm{d}}\right) N z \mid z \in \mathbb{R}^{\operatorname{coldim}(N)}\}$.

It is characterized by the particular response $w_{\mathrm{r}, 0}$ and a subspace-the column span of the matrix $\mathscr{H}_{T_{\mathrm{r}}}\left(w_{\mathrm{d}}\right) N$. Algorithm 1 summarizes the necessary steps for data-driven computation of $\mathscr{W}_{\mathrm{r}}$ from $w_{\mathrm{d}}, R$, and $r_{\mathrm{r}}$.

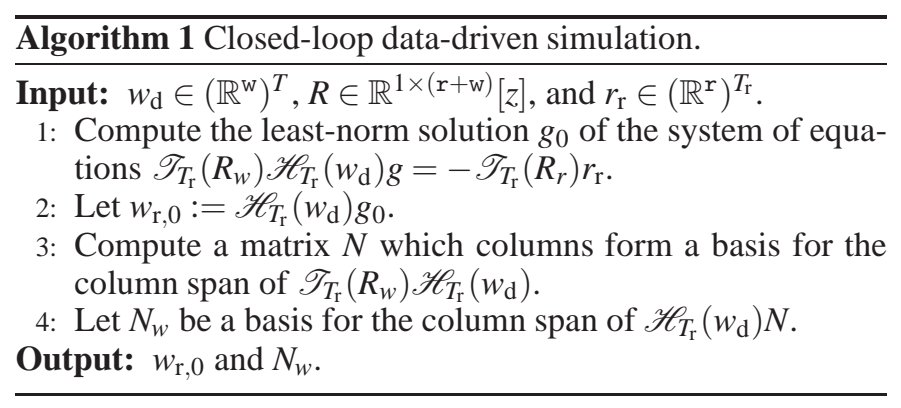

We have shown that under assumptions 1 and 2, the set

$$
\mathscr{W}_{\mathrm{r}}:=\left\{w_{\mathrm{r}, 0}+N_{w} z \mid z \in \mathbb{R}^{\operatorname{coldim}\left(N_{w}\right)}\right\} .
$$

computed by Algorithm 1 is equal to the set of $T_{\mathrm{r}}$ samples long responses of the closed-loop system $\mathscr{B}_{\mathscr{C}}$ to the reference signal $r_{\mathrm{r}}$, i.e.,

$$
\mathscr{W}_{\mathrm{r}}=\left\{w \in\left(\mathbb{R}^{\mathrm{w}}\right)^{T_{\mathrm{r}}}\left|\left(r_{\mathrm{r}}, w\right) \in \mathscr{B}_{\mathscr{C}}\right|_{T_{\mathrm{r}}}\right\} .
$$

\section{Simulation example}

The data $w_{\mathrm{d}}=\left(u_{\mathrm{d}}, y_{\mathrm{d}}\right)$, used for the closed-loop data driven simulation, is the first 10 samples from the step response of a randomly generated first order system $\mathscr{B}$ interconnected with the controller $\mathscr{C}_{1}:=\{(r, u, y) \mid u=-y\}$. The aim is to compute the first $T_{\mathrm{r}}=10$ samples of the step response of $\mathscr{B}_{\mathscr{C}_{2}}$, where $\mathscr{C}_{2}:=\{(r, u, y) \mid u=y\}$. For this purpose we use Algorithm 1 , i.e., we do not compute explicitly a representation of $\mathscr{B}$. Note that either of the systems $\mathscr{B}_{\mathscr{C}_{1}}, \mathscr{B}_{\mathscr{C}_{2}}$ can be unstable.

In order to ensure that assumption 2 is satisfied, we augment the given trajectory - the step response of $\mathscr{B}_{\mathscr{C}_{1}}$ - with $T_{\mathrm{r}}$ zeros. This takes into account the zero initial conditions of the given trajectory and ensures that the initial conditions of the computed response $r_{\mathrm{r}}$ of $\mathscr{B}_{\mathscr{C}_{1}}$ are also zero (i.e., $N_{w}=0$ ). The results for a particular system $\mathscr{B}$ are shown in Figure 1 . We verify that up to numerical errors $r_{\mathrm{r}}$ matches the step response $s$ of $\mathscr{B}_{\mathscr{C}_{2}}$, obtained by model-based simulation. A MATLAB file reproducing the simulation result is available from:

http://users.ecs.soton.ac.uk/im/test_cdds.m
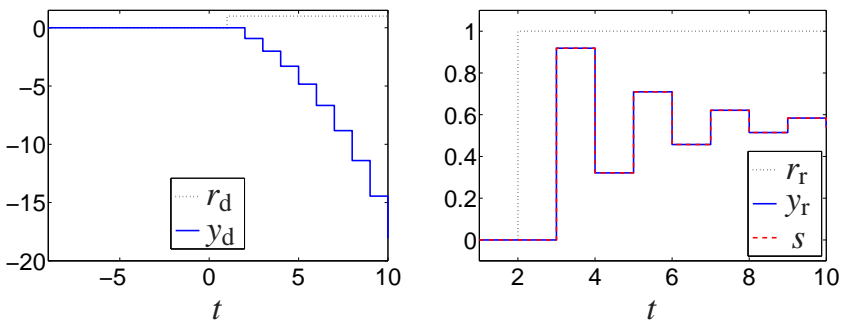

Fig. 1. Step responses of $\mathscr{B}_{\mathscr{C}_{1}}$ (left) and $\mathscr{B}_{\mathscr{C}_{2}}$ (right).

\section{REFERENCES}

I. Markovsky and P. Rapisarda. On the linear quadratic datadriven control. In Proceedings of the European Control Conference, pages 5313-5318, Kos, Greece, 2007.

I. Markovsky and P. Rapisarda. Data-driven simulation and control. Int. J. Control, 81(12):1946-1959, 2008.

I. Markovsky, J. C. Willems, P. Rapisarda, and B. De Moor. Data driven simulation with applications to system identification. In Proceedings of the 16th IFAC World Congress, Prague, Czech Republic, 2005.

I. Markovsky, J. C. Willems, S. Van Huffel, and B. De Moor. Exact and Approximate Modeling of Linear Systems: A Behavioral Approach. SIAM, March 2006.

P. Van Overschee and B. De Moor. Subspace Identification for Linear Systems: Theory, Implementation, Applications. Kluwer, Boston, 1996.

J. C. Willems. From time series to linear system-Part I. Finite dimensional linear time invariant systems. Automatica, 22 (5):561-580, 1986.

J. C. Willems, P. Rapisarda, I. Markovsky, and B. De Moor. A note on persistency of excitation. Control Lett., 54(4):325329, 2005. 